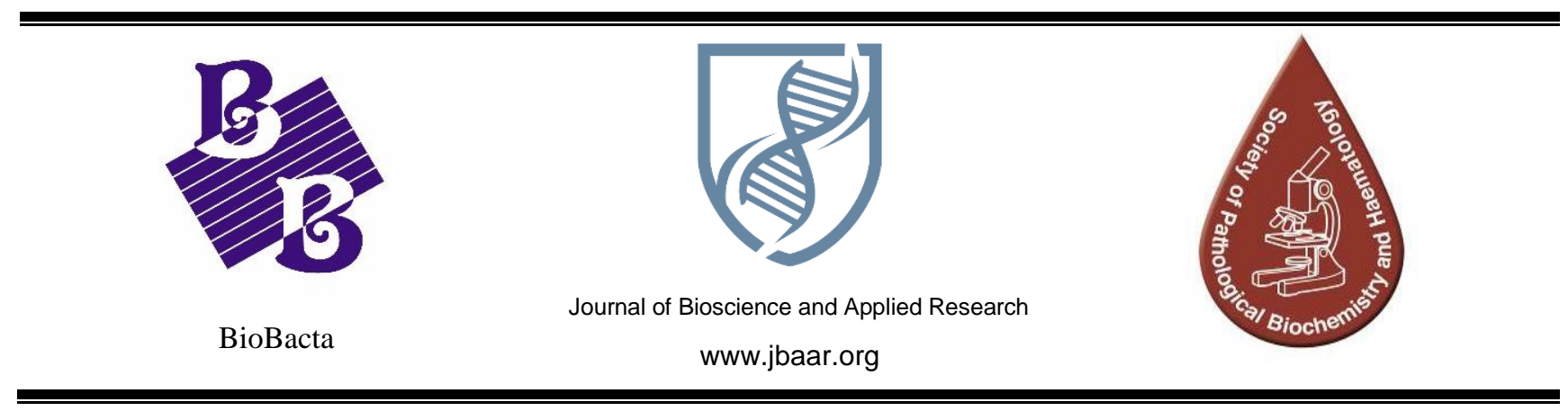

\title{
Ferritin as a biomarker of infection in COVID-19 non-hospitalized patients
}

\author{
Ahmed Abdelhalim Yameny \\ Society of Pathological Biochemistry and Hematology, Egypt \\ Ahmed A. Yameny (Email: dr.ahmedyameny@yahoo.com) \\ Tel: (002)01222708665, (002)01146051753
}

\section{ABSTRACT}

DOI: 10.21608/jbaar.2021.172371

Background: Serum ferritin is an iron storage protein with a primary role of regulating cellular oxygen metabolism, Ferritin has emerged as a key in the immune system, and its role as an acute phase reactant, recent studies have focused on the role of serum inflammatory markers that predict COVID-19. Patients and methods: This study included 210 non hospitalized patients with confirmed COVID-19 infection in Alexandria, Egypt, these study subjects were randomly selected irrespective of the age group and both genders were included, Assay procedure as manufactory instructions and Reagents of PerkinElmer Health sciences. Inc USA kit human ferritin enzyme immunoassay test catalog number 10601 was used. Results: The present study included patients aged from14 years to 75 years mean age was $44.5 \pm 30.5$ who were confirmed to have Covid-19 based on real-time reverse transcription-polymerase chain reaction, the female gender was more frequent $(n=132,62.9 \%)$ than Male gender $(n=78,37.1 \%)$. This study reveals high serum ferritin level in 150 patients (71.4\%), which was a significant biomarker for COVID-19 diagnosis in nonhospitalized patients (Outpatients and patients under home observation), the $p$-value is .014738 . Significant at $p<.05$. Conclusion and Recommendations: Serum ferritin level is a good biomarker of infection in COVID-19 non-hospitalized patients, many other studies revealed ferritin is a good marker for COVD-19 hospitalized patients, Increased ferritin levels could cause a cytokine storm by exerting direct immunosuppressive and pro-inflammatory effects, It has been reported that fatal outcomes by COVID-19 are accompanied by cytokine storm syndrome, Many individuals with diabetes and immunosuppressive diseases exhibit elevated serum ferritin levels, and it is known that they face a higher probability to experience serious complications from COVID-19 infection, so these patients must go to the medical laboratory for serum ferritin level analysis, to decrease ferritin levels might be the treatment with iron chelators. Deferoxamine, which was approved by the FDA, with decreasing dietary iron should be also considered as they have been shown to modify serum ferritin levels.

Keywords: Ferritin, COVID-19, non-hospitalized patients, biomarker, hyperferritinemia 


\section{Introduction}

A new type of pneumonia defined as coronavirus disease 2019 (COVID-19) has been widely spreading in China in December 2019, and even in many other countries ${ }^{(1)}$. World Health Organization (WHO) identified COVID-19 as a new pandemic in March 2020, the outbreak spread to 187 countries worldwide within 3 months with high morbidity and mortality ${ }^{(2)} .80 \%$ of patients infected by COVID-19 may be asymptomatic or only mildly symptomatic, but around $10 \%$ develop severe respiratory symptoms that evolve to acute respiratory distress syndrome (ARDS) $)^{(3)}$, a virus with a single-stranded RNA genome and characteristic surface spike proteins. Same as other SARS-like viruses ${ }^{(4)}$. COVID-19 patients admitted to ICUs have been found to have higher concentrations of proinflammatory cytokines and, importantly, increased secretion of those T-helper-2 (Th2) cytokines suppressing inflammation ${ }^{(5)}$.

Serum ferritin is an iron storage protein with the primary role of regulating cellular oxygen metabolism. Ferritin has emerged as a key in the immune system, and its role as an acute phase reactant, recent studies have focused on the role of serum inflammatory markers that predict COVID$19^{(6)}$. Increased ferritin levels could cause a cytokine storm by exerting direct immunosuppressive and pro-inflammatory effects ${ }^{(7)}$. The levels of ferritin, a crucial immune response mediator, increase in severe Covid-19 cases $^{(7)}$. Many individuals with diabetes exhibit elevated serum ferritin levels so that ferritin levels might be a crucial factor influencing COVID-19 severity ${ }^{(7,8)}$.

In light of these findings, and considering the role of hyperferritinemia, via direct immune-suppressive and pro-inflammatory effects, this study aimed to evaluate the use of ferritin as a biomarker in COVID-19 Outpatients and patients under home observation.

\section{Patients and methods}

\subsection{Study population Patients}

This study included 210 non hospitalized patients with confirmed COVID-19 infection, these study subjects were randomly selected irrespective of the age group and both genders were included.

It was performed following the ethical standards laid down in the 1964 Declaration of Helsinki and its later amendments. All the studied population was informed about the purpose of sample collection and their consents were obtained. Patients were free to refuse sample collection.

\subsection{Data collection}

In this cross-sectional study, we obtained data regarding 210 non hospitalized patients with confirmed COVID-19 via real-time reverse transcription-polymerase chain reaction (PCR), they came to Alyameny laboratory in Alexandria, Egypt for biomarkers and complete blood count investigations, We reviewed the medical records and compiled data between August 12 and December 30, 2020.

\subsection{Collection and processing of blood samples:}

$1 \mathrm{ml}$ blood sample was collected for performing serum ferritin test (PerkinElmer Health sciences. Inc USA) on 120 Positive COVID-19 patients for individuals matching in age and gender.

\subsection{Assay procedure as manufactory instructions:}

Reagents of PerkinElmer Health sciences. Inc USA kit human ferritin enzyme immunoassay test catalog number 10601 and samples allowed to be at room temperature before testing, (serum was separated from a blood sample by centrifugation), considered normal serum ferritin level for adult males 16-220 $\mathrm{ng} / \mathrm{ml}$, for adult females $10-124 \mathrm{ng} / \mathrm{ml}$ and children (6 months- 15 years) $7-140 \mathrm{ng} / \mathrm{ml}$

\subsection{Statistical analysis}

Data were analyzed using SPSS statistical software, version 20.0(SPSS, Chicago, Illinois, USA). All continuous data are presented as means and standard deviations, while categorical data are presented as 
numbers and percentages. A chi-square test was used to compare categorical variables. Multivariate regression analysis was performed to analyze relationships between COVID-19 infected patients and serum ferritin level, this model was generated using independent variables achieving a p-value of 0.10 during

bivariate analysis. Then, the best-fit model was generated without interaction variables. For all calculations, a p-value of less than 0.05 was considered statistically significant.

\section{Results:}

Table (1): shows The percentage of COVID-19 non hospitalized Patients relation to the serum ferritin level detected by the ELISA technique, The present study included patients aged from 14 years to 75 years mean age was $44.5 \pm 30.5$ who were confirmed to have Covid-19 based on real-time reverse transcription-polymerase chain reaction, female gender was more frequent $(n=132,62.9 \%)$ than Male gender $(n=78,37.1 \%)$. This study reveals high serum ferritin levels in 150 patients $(71.4 \%)$, which was a significant biomarker for COVID-19 diagnosis in non-hospitalized patients(Outpatients and patients under home observation).

Table (1): The percentage of COVID-19 non-hospitalized Patients relation to the serum ferritin level detected by the ELISA technique.

\begin{tabular}{|c|c|c|c|c|c|c||}
\hline \multirow{2}{*}{} & \multicolumn{3}{|c|}{$\begin{array}{c}\text { Serum ferritin level } \\
\text { By ELISA }\end{array}$} & \multicolumn{2}{c|}{} \\
\cline { 2 - 7 } $\begin{array}{c}\text { COVID-19 } \\
\text { POSITIVE } \\
\text { Patients }\end{array}$ & \multicolumn{2}{|c|}{ *ormal } & **High level & \multicolumn{2}{c|}{ Total } \\
\cline { 2 - 7 } & No. & $\%$ & No. & $\%$ & No. & $\%$ \\
\hline Male & 30 & 50 & 48 & 32 & 78 & 37.1 \\
\hline Female & 30 & 50 & 102 & 68 & 132 & 62.9 \\
\hline Total & 60 & & 150 & & 210 & \\
\hline
\end{tabular}

The chi-square statistic is 5.9476. The $p$-value is .014738. Significant at $p<.05$.

The chi-square statistic with Yates correction is 5.2016. The $p$-value is .022567. Significant at $p<.05$. 
Figure (1): Serum ferritin level with the percentage of COVID-19 PATIENTS

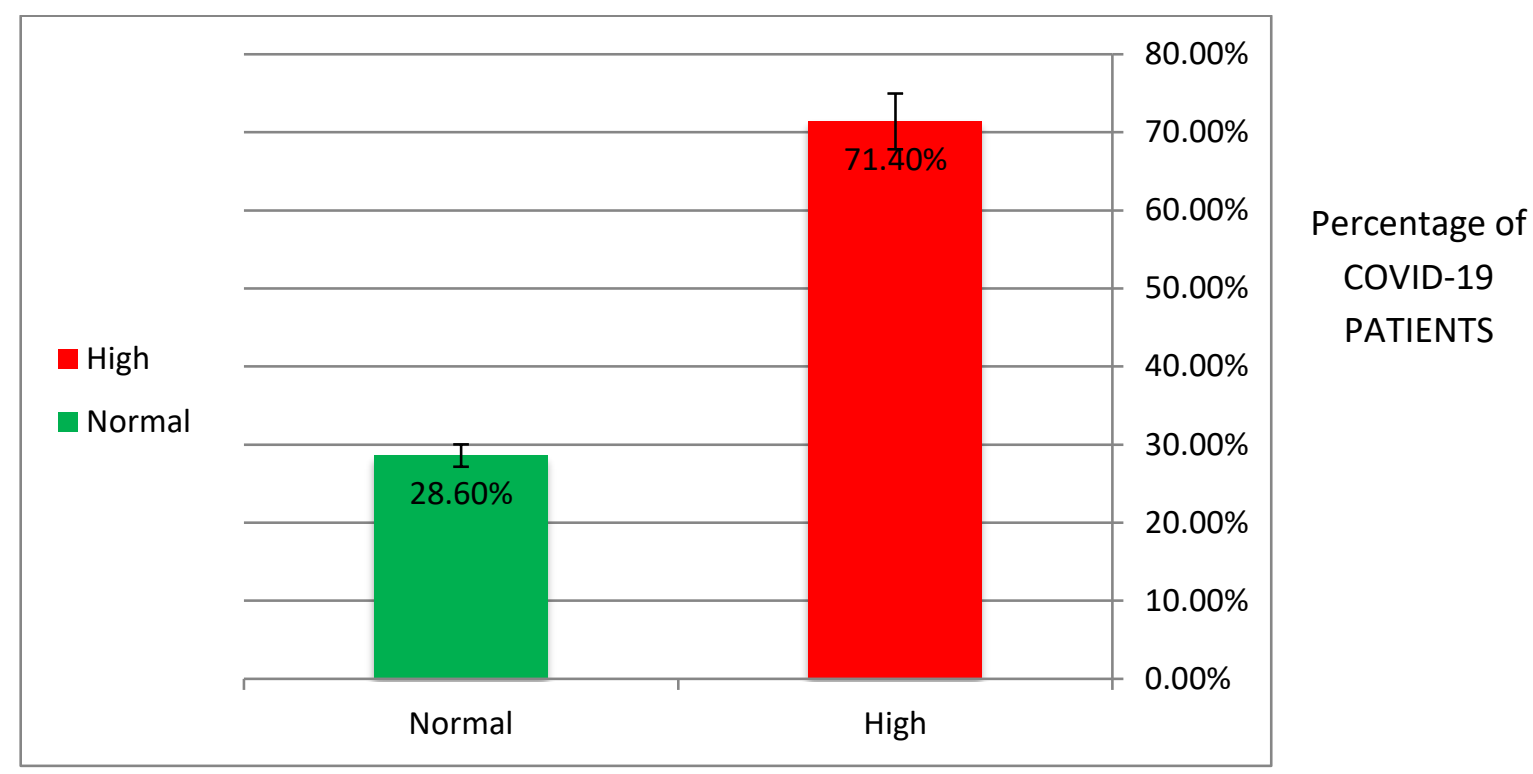

Serum ferritin level $(\mathrm{ng} / \mathrm{ml})$

\section{Discussion:}

COVID-19 has non-specific disease symptoms, and false-negative and delayed polymerase chain reaction results, so additional markers are needed for the diagnosis and surveillance of the disease ${ }^{(9)}$, The early diagnosis of Covid-19, and risk complications of the disease may reduce morbidity and mortality rates $^{(10)}$.

Ferritin is a large protein $(440 \mathrm{kDa})$ present within the cytosol, or less often, within the mitochondria of the cell, and it can sequester up to approximately 4500 atoms of iron ${ }^{(11)}$, In plasma, ferritin circulates as apoferritin, a non-iron containing molecule. The plasma level generally reflects overall iron storage, with $1 \mathrm{ng}$ of ferritin per $\mathrm{ml}$ indicating approximately $10 \mathrm{mg}$ of total iron stores ${ }^{(12)}$.

Pathways related to inflammation and autoimmunity impacted ferritin regulation, which affects ferritin content within cells, among these pathways, several pro-inflammatory cytokines ${ }^{(\mathbf{1 3})}$,
It has been reported that fatal outcomes by COVID19 are accompanied by cytokine storm syndrome, thereby it has been suggested that it cause disease severity ${ }^{(5)}$, Many individuals with diabetes exhibit elevated serum ferritin levels ${ }^{(14)}$, and it is known that they face a higher probability to experience serious complications from COVID-19(8).

210 Outpatients with mild symptoms and patients under home observation were included in this study, with confirmed COVID-19 infection, it showed elevation of serum ferritin level in 150 patients from 210 with a percentage of $71.4 \%$ which has a p-value of 0.0147 , It was a significant value. The present study included patients aged from14 years to 75 years mean age was $44.5 \pm 30.5$ who were confirmed to have Covid-19 based on real-time reverse transcription-polymerase chain reaction (PCR), Reagents of Chemux Bioscience. Inc USA kit human ferritin enzyme immunoassay test (ELISA) was used in this study.

Many studies were conducted on severe and very severe hospitalized patients, COVID-19 showed data 
consistent with cytokine storm involving elevated inflammatory markers, including ferritin, which has been associated with critical and life-threatening illness, a study involving 20 patients with Covid-19 evaluated ferritin, a serum inflammatory marker, and reported increased serum ferritin levels in patients with severe and very severe Covid-19, with the levels being significantly higher in the very severe $\operatorname{group}^{(15)}$,

Also, Chen et al. analyzed the clinical characteristics of 99 patients, in which 63 of them had serum ferritin way above the normal range ${ }^{(16)}$, Elevated ferritin levels were found also in autopsies of 12 patients whose cause of death was SARSCoV-2 infection ${ }^{(17)}$. Another study involving 69 patients with severe Covid-19 reported significantly higher ferritin levels in patients with severe disease than in those with nonsevere disease ${ }^{(18)}$.

A possible strategy to decrease ferritin levels might be the treatment with iron chelators. Deferoxamine may be a good candidate since is a non-toxic iron chelator clinically approved by the $\mathrm{FDA}^{(19)}$ with decreasing dietary iron should be also considered as they have been shown to modify serum ferritin levels ${ }^{(20)}$.

\section{Conflict of interest}

There are no conflicts of interest.

\section{Financial support and sponsorship}

This research did not receive any specific grant from funding agencies in the public, commercial, or not-for-profit sectors.

\section{References}

1. Zhu N, Zhang D, Wang W, Li X, Yang B, Song $\mathrm{J}$, et al. A novel coronavirus from patients with pneumonia in China, 2019. N Engl J Med 2020. DOI: 10.1056/NEJMoa2001017.

2. Connors JM, Levy JH. COVID-19 and its implications for thrombosis and anticoagulation. Blood. 2020;135(23):20332040.
3. Marietta M, Ageno W, Artoni A, De Candia E, Gresele P, Marchetti M, et al. COVID-19 and haemostasis: a position paper from Italian Society on Thrombosis and Haemostasis (SISET). Blood Transfus. 2020 May; 18(3):167-9.

4. Harenberg J, Favaloro E. COVID-19: progression of disease and intravascular coagulation - present status and future perspectives. Clin Chem Lab Med. 2020;58(7):1029-1036. doi:10.1515/cclm2020- 0502.

5. Huang $\mathrm{C}$, Wang $\mathrm{Y}$, Li $\mathrm{X}$, et al. Clinical features of patients infected with 2019 novel coronavirus in Wuhan. China. Lancet. 2020;395:497-506.

6. Yang Z, Shi J, He Z, Lü Y, Xu Q, Ye C et al. Predictors for imaging progression on chest CT from coronavirus disease 2019 (COVID-19) patients. Aging (Albany NY) 2020;12:6037-48.

7. Vargas-Vargas M, Cortés-Rojo C. Ferritin levels and COVID-19. Rev Panam Salud Publica2020;44:e72.

8. American Diabetes Association. How COVID19 Impacts People with Diabetes. Available at: https://www.diabetes.org/coronavirus-covid19/how-coronavirus-impacts-people-withdiabetes Accessed May 22,2020.

9. Lechien JR, Chiesa-Estomba CM, De Siati DR, Horoi M, Le Bon SD, Rodriguez A, et al. Olfactory and gustatory dysfunctions as a clinical presentation of mild-to-moderate forms of the coronavirus disease

(COVID-19): a multicenter European study. Eur Arch Otorhinolaryngol 2020;277:2251-61.

10. Li K, Wu J, Wu F, Guo D, Chen L, Fang Z, et al. The clinical and chest CT features associated with severe and critical COVID-19 pneumonia. Invest Radiol 2020;55:327-31.

11. Harrison PM, Arosio P. The ferritins: molecular properties, iron storage function and cellular 
regulation. Biochim Biophys Acta BBABioenerg 1996; 1275(3):161-203.

12. Finch CA, Stray S, Huebers HA, et al. Plasma ferritin determination as a diagnostic tool. West J Med 1986; 145(5):657.

13. Torti FM, Torti SV. Regulation of ferritin genes and protein. Blood J Am Soc Hematol 2002; 99(10):3505-16.

14. Khalil UA, Seliem FO, Alnahal A, Awad M, Sadek AM, Fawzy MS. Association of serum ferritin with insulin resistance in offsprings of type 2 diabetes. Egypt $\mathrm{J}$ Intern Med. 2018;30:13-7.

15. Zhou B, She J, Wang Y, Ma X. Utility of Ferritin, Procalcitonin, and C-reactive Protein in Severe Patients with 2019 Novel Coronavirus Disease. In: https://doi.org/10.21203/rs.3.rs18079/v1 [22 May 2020].

16. Chen N, Zhou M, Dong X, Qu J, Gong F, Han $\mathrm{Y}$, et al. Epidemiological and clinical characteristics of 99 cases of 2019 novel coronavirus pneumonia in Wuhan, China: a descriptive study. Lancet. 2020;395(10223):507-13.

17. Fox SE, Akmatbekov A, Harbert JL, Li G, Brown JQ, Vander Heide RS. Pulmonary and Cardiac Pathology in Covid-19: The First Autopsy Series from New Orleans. medRxiv 2020.04.06-20050575; DOI: https://doi.org/10.1101/2020.04.06.20050575.

18. Liu T, Zhang J, Yang Y, Ma H, Li Z, Zhang J, et al. The role of interleukin- 6 in monitoring severe cases of coronavirus disease 2019. EMBO Mol Med2020;12:e12421.

19. Mobarra N, Shanaki M, Ehteram H, Nasiri H, Sahmani M, Saeidi M, et al. A Review on Iron Chelators in Treatment of Iron Overload Syndromes. Int J Hematol Oncol Stem Cell Res. 2016;10(4):239-47.

20. Fleming DJ, Tucker KL, Jacques PF, Dallal GE, Wilson PWF, Wood RJ. Dietary factors associated with the risk of high iron stores in the elderly Framingham Heart Study cohort. Am J Clin Nutr. 2002;76:1375-84. 